\title{
Estudo Histopatológico do Efeito do Tenoxicam com Água Bidestilada ou com Cloreto de Sódio a 0,9\% no Endotélio Venoso de Coelhos *
}

\section{Histopathologic Study on the Effects of Tenoxicam with Bidistilled Water or with $0.9 \%$ Sodium Chloride in Rabbits Venous Endothelium}

Taylor Brandão Schnaider, TSA ${ }^{1}$, Alcino Lázaro da Silva ${ }^{2}$, Míriam de Fátima Brasil Engelman ${ }^{3}$, Yara Juliano ${ }^{4}$, Neil Ferreira Novo ${ }^{5}$, Gabrielle Sormanti Schnaider ${ }^{6}$, Caroline Sormanti Schnaider ${ }^{7}$

\section{RESUMO}

Schnaider TB, Silva AL, Engelman MFB, Juliano Y, Novo NF, Schnaider GS, Schnaider CS - Estudo Histopatológico do Efeito do Tenoxicam com Água Bidestilada ou com Cloreto de Sódio a $0,9 \%$ no Endotélio Venoso de Coelhos

Justificativa e Objetivos - Em estudo com células endoteliais de veias umbilicais humanas expostas à indometacina, foi observado aumento da atividade pró-coagulante. Estudo em coelhos comprovou a presença de trombose nas veias auriculares após administração de tenoxicam com seu diluente ou de seu diluente isolado. Não foram encontrados estudos na literatura consultada que tenham avaliado o endotélio venoso após a administração do tenoxicam, em seres humanos. 0 objetivo desta pesquisa foi avaliar se o tenoxicam com cloreto de sódio a 0,9\% ( $\mathrm{NaCl}$ a 0,9\%) provoca alterações no endotélio venoso de coelhos, como as observadas quando associado ao seu diluente (água bidestilada).

Método - Noventa coelhos (2.000 - $3.500 \mathrm{~g})$ foram distribuídos aleatoriamente em dois grupos: Controle, com administração de $\mathrm{NaCl}$ a 0,9\%; Experimento, com tenoxicam (20 mg) associado à água bidestilada ou ao $\mathrm{NaCl}$ a $0,9 \%$. O volume injetado nos dois grupos foi constante de $2 \mathrm{ml}$. A anestesia foi induzida com maleato de acepromazina, cloridrato de cetamina e cloridrato de xilazina, sendo a punção das veias auriculares caudais direita e esquerda realizada com agulha tipo borboleta 27G. Os animais foram mantidos no biotério por 6 $h$, $12 h$ e $24 h$, novamente anestesiados e submetidos à

\footnotetext{
* Recebido da (Received from) Faculdade de Medicina de Pouso Alegre, MG. Doutorado realizado no Curso de Pós-Graduação em Técnica Cirúrgica e Cirurgia Experimental da Faculdade de Medicina da Universidade Federal de Minas Gerais (UFMG).

1. Professor Titular do Departamento de Clínica Cirúrgica da Faculdade de Medicina - Universidade do Vale do Sapucaí (UNIVAS).

2. Professor Titular da Disciplina de Cirurgia do Aparelho Digestivo da Faculdade de Medicina - Universidade Federal de Minas Gerais (UFMG).

3. Professora Adjunta do Departamento de Ciências Fisio-morfológicas e Patologia da Faculdade de Medicina (UNIVAS).

4. Professora Adjunta da Disciplina de Bioestatística da Escola Paulista de Medicina - Universidade Federal do Estado de São Paulo (UNIFESP).

5. Professor Adjunto da Disciplina de Bioestatística da Escola Paulista de Medicina (UNIFESP).

6. ME de Pediatria (UNICAMP).

7. Doutoranda da Faculdade de Medicina (UNIVAS)

Apresentado (Submitted) em 19 de julho de 2001

Aceito (Accepted) para publicação em 08 de outubro de 2001

Correspondência para (Mail to):

Dr. Taylor Brandão Schnaider

Av. Francisca R. de Paula, 289

37550-000 Pouso Alegre, MG

E-mail: sormanti@uai.com.br
}

(C) Sociedade Brasileira de Anestesiologia, 2002 eutanásia, sendo então realizada exérese das aurículas em sua base e posterior avaliação microscópica das veias.

Resultados - Observou-se trombose no grupo Experimento, numa porcentagem de $19,4 \%$ após administração do tenoxicam com água bidestilada e 22,2\% após administração do tenoxicam com $\mathrm{NaCl}$ a $0,9 \%$. No grupo Controle, em que foi injetado somente $\mathrm{NaCl}$ a 0,9\%, nenhuma das veias apresentou trombose.

Conclusões - Os resultados encontrados permitem concluir que o tenoxicam, com água bidestilada ou com solução de cloreto de sódio a 0,9\%, produziu trombose nas veias em que foi injetado.

UNITERMOS: ANALGÉSICOS, Antiinflamatório: tenoxicam; ANIMAL: coelho

\section{SUMMARY}

Schnaider TB, Silva AL, Engelman MFB, Juliano Y, Novo NF, Schnaider GS, Schnaider CS - Histopathologic Study on the Effects of Tenoxicam with Bidistilled Water or with $0.9 \%$ Sodium Chloride in Rabbits Venous Endothelium

Background and Objectives - After exposure to indomethacin, human umbilical vein endothelial cells have shown increased procoagulant activity. Thrombosis in rabbit auricular veins has been observed after injection of tenoxicam with its diluent or of the diluent alone ${ }^{2}$. Human studies evaluating venous endothelium after tenoxican injection were not found in the literature. This study aimed at evaluating whether tenoxicam with $0.9 \%$ sodium chloride changes the venous endothelium of rabbits as it is observed when associated to its diluent (bidistilled water).

Methods - The study involved 90 rabbits (2000 and 3500 grams) divided in two groups: Control group, which received $0.9 \% \mathrm{NaCl}$; Experimental group, which received tenoxicam $(20 \mathrm{mg})$ with bidistilled water or with $0.9 \% \mathrm{NaCl}$. A constant volume of $2 \mathrm{ml}$ was administered to both groups. Anesthesia was induced with acepromazine maleate, ketamine hydrochloride and xylazine hydrochloride, and the puncture of right and left caudal auricular veins was performed with a $27 \mathrm{G}$ butterfly needle. Animals were confined for $6 \mathrm{hrs}, 12 \mathrm{hrs}$, and $24 \mathrm{hrs}$, when they were once more anesthetized and sacrificed, with extraction of the auriculae at their base, followed by microscopic venous study.

Results - Thrombosis was observed in $19.4 \%$ of the Experimental Group after the administration of tenoxicam with bidistilled water and in $22.2 \%$ after the administration of tenoxicam with $0.9 \%$ sodium chloride. In the Control group, which has only received $0.9 \%$ sodium chloride, no thrombosis was observed.

Conclusions - It was possible to conclude that tenoxicam, either with bidistilled water or $0.9 \%$ sodium chloride, has induced thrombosis in the veins it was injected.

KEY WORDS - ANALGESICS, Anti-inflammatory: tenoxicam; ANIMAL: rabbit 


\section{INTRODUÇÃO}

$\mathrm{E}_{\mathrm{s}}^{\mathrm{m}}$ m virtude de, na nossa clínica anestesiológica, terem sido encontrados pacientes que relataram sinais e sintomas de vasculite (dor, calor e rubor), após administração venosa direta do tenoxicam com água bidestilada (seu diluente comercial), pesquisamos e, na literatura consultada, verificamos a inexistência de estudos relativos a essa ocorrência em seres humanos.

Nygard e col., em decorrência da associação entre drogas antiinflamatórias não-esteróides e lesão microvascular observada no intestino, pesquisaram as alterações que ocorrem durante a coagulação, em culturas com células endoteliais de veias umbilicais humanas (HUVEC), quando expostas à indometacina, a lipopolissacarídeo bacteriano ou à associação de ambos, nos tempos de $1 \mathrm{~h}, 6 \mathrm{~h}, 12 \mathrm{~h}$ e $24 \mathrm{~h}$. Os autores obtiveram os seguintes resultados: o lipopolissacarídeo induziu significante manifestação de atividade pró-coagulante às $6 \mathrm{~h}, 12 \mathrm{~h}$ e $24 \mathrm{~h}$, com um significante aumento da produção de 6-keto-PGF ${ }_{1 \alpha}$, enquanto que o aumento da produção de $\mathrm{PGE}_{2}$ foi muito menos pronunciado; quando a incubação continha indometacina sozinha, esta induziu a uma resposta de atividade pró-coagulante tempo-dependente; quando a incubação continha indometacina e lipopolissacarídeo, a resposta da atividade pró-coagulante era muito maior que a produzida pelas culturas contendo indometacina ou lipopolissacarídeo isolados ${ }^{1}$.

Schnaider e col., em estudos experimentais em coelhos, avaliaram pela histopatologia a presença ou a ausência de trombose após administração do tenoxicam com seu diluente comercial e do seu diluente isolado, no endotélio venoso. Os autores constataram a ocorrência de 19,4\% de trombose, em ambos os casos ${ }^{2}$. Desse estudo, restou uma dúvida: se a trombose foi provocada pelo tenoxicam ou pela água bidestilada usada como diluente.

O objetivo desta pesquisa foi avaliar, pela histopatologia, se o tenoxicam com $\mathrm{NaCl}$ a $0,9 \%$ provoca alterações no endotélio venoso de coelhos, como as observadas quando este antiinflamatório é associado à água bidestilada.

\section{MÉTODO}

Após aprovação do Comitê de Ética em Pesquisa Animal, 90 coelhos (Oryctolagus cuniculus), brancos, da linhagem Nova Zelândia, machos, com idade acima de 10 semanas, com peso variando entre 2.000 e 3.500 gramas, foram distribuídos aleatoriamente em dois grupos: Experimento e Controle. O grupo Experimento foi subdividido em 3 subgrupos de 24 coelhos cada um, denominados subgrupos E6, E12 e E24, que corresponderam respectivamente aos períodos de observação de $6 \mathrm{~h}, 12 \mathrm{~h}$ e $24 \mathrm{~h}$, tendo sido administrados aleatoriamente, nas veias auriculares caudais direita ou esquerda de cada animal, tenoxicam/diluente ou tenoxicam $/ \mathrm{NaCl}$ a $0,9 \%$. O grupo Controle foi subdividido também em 3 subgrupos de 6 coelhos cada um, denominados subgrupos $\mathrm{C} 6, \mathrm{C} 12$ e C24, que corresponderam respectivamente aos períodos de observação de 6 h, 12 h e 24 h, tendo sido administrado $\mathrm{NaCl}$ a $0,9 \%$ nas veias auriculares caudais direita e esquerda de cada animal.

Os animais não sofreram quaisquer restrições com relação à ingestão de alimentos e água até o momento do experimento, sendo então pesados e anestesiados com maleato de acepromazina a $1 \%$ na dose de $2 \mathrm{mg} \cdot \mathrm{kg}^{-1}$, cloridrato de cetamina na dose de $35 \mathrm{mg} \cdot \mathrm{kg}^{-1}$ e cloridrato de xilazina a $2 \%$ na dose de 5 $\mathrm{mg} \cdot \mathrm{kg}^{-1}$, por via muscular na região glútea.

Passados 10 minutos, após administração da anestesia, cada animal foi posicionado em decúbito dorsal sobre a mesa operatória onde os membros torácicos e pélvicos foram contidos com barbantes às extremidades da mesa.

Transcorridos 15 minutos, foi testado o plano anestésico, pesquisando-se o reflexo da resposta do membro pélvico direito ao estímulo doloroso na prega cutânea entre os dígitos II e III. Em seguida, foi realizada a anti-sepsia com álcool a 70\% e colocados os campos operatórios esterilizados. Foram puncionadas as veias auriculares caudais direita e esquerda com agulha tipo borboleta $27 \mathrm{G}$. Foram injetados, aleatoriamente, tenoxicam/diluente ou tenoxicam $/ \mathrm{NaCl}$ a $0,9 \%$ no grupo Experimento e $\mathrm{NaCl}$ a $0,9 \%$ no grupo Controle (Figura 1). A seguir, foram demarcados os locais de punção com caneta "Texta fine line 700". Os $2 \mathrm{ml}$ das substâncias foram acondicionados previamente em seringas de $3 \mathrm{ml}$ e injetados na velocidade de $1 \mathrm{ml}$ por 30 segundos.

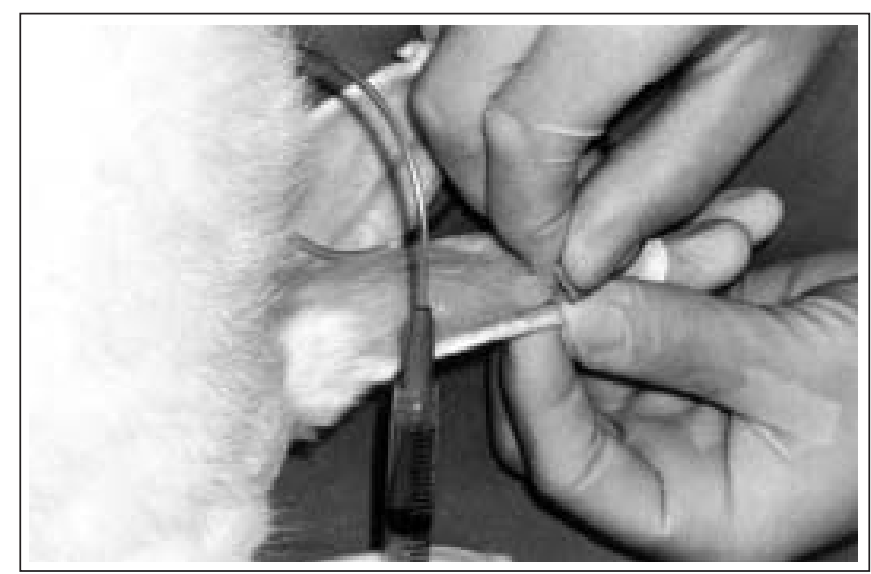

Figura 1 - Punção da Veia Auricular Caudal com Agulha 27G (tipo borboleta), para Administração dos Fármacos

Transcorrido o tempo de evolução programado para cada subgrupo ( 6 h, 12 h ou 24 h), os animais foram novamente anestesiados, pela mesma técnica utilizada no início do experimento e submetidos à eutanásia, com injeção intracardíaca de $3 \mathrm{ml}$ de cloreto de potássio a 10\%. A seguir, foi feito um corte triangular na parte rostral das aurículas e colocado um ponto, com fio de algodão 000 , na parte distal da aurícula direita. Procedeu-se, então, a exérese das aurículas em sua base, as quais foram fixadas em formalina neutra tamponada a $10 \%$, após observação macroscópica das mesmas 
(Figura 2). Os recipientes contendo as peças cirúrgicas foram identificados com o grupo, subgrupo e o número do respectivo animal. As peças foram incluídas em parafina, sendo realizados 5 cortes com 5 micrômetros de espessura cada, a partir de $1 \mathrm{~cm}$ do local em que foi realizada a punção, em direção à parte proximal da aurícula, utilizando-se micrótomo rotativo com navalha descartável (Leica RM-2025). A seguir, os cortes foram colocados em lâminas, corados por hematoxilina-eosina $(\mathrm{H}-\mathrm{E})$ e cobertos por lamínulas. As lâminas foram analisadas ao microscópio óptico (Lambda LQT-2) com aumentos originais de 40, 100 e 400 vezes, digitalizadas (Câmera Samsung SHC-410 NAD) e gravadas em disquetes de $3 \frac{1}{2}$ polegadas.

Para análise estatística dos resultados foi aplicado o teste da partição do Qui-quadrado para tabelas $2 \times \mathrm{N}$, com o objetivo de comparar, separadamente para cada grupo, os períodos de eutanásia de 6 h, 12 h e 24 h, em relação às presenças de trombose. O mesmo teste foi aplicado para comparar as presenças do total de trombose dos grupos Controle, Tenoxicam/Diluente e Tenoxicam/ $\mathrm{NaCl}$ a $0,9 \%$, independente do período de eutanásia. Em todos os testes fixou-se em 0,05 ou $5 \%(\alpha \leq 0,05)$ o nível para a rejeição da hipótese de nulidade, assinalando-se com um asterisco $\left(^{*}\right)$ e em negrito os valores significantes.

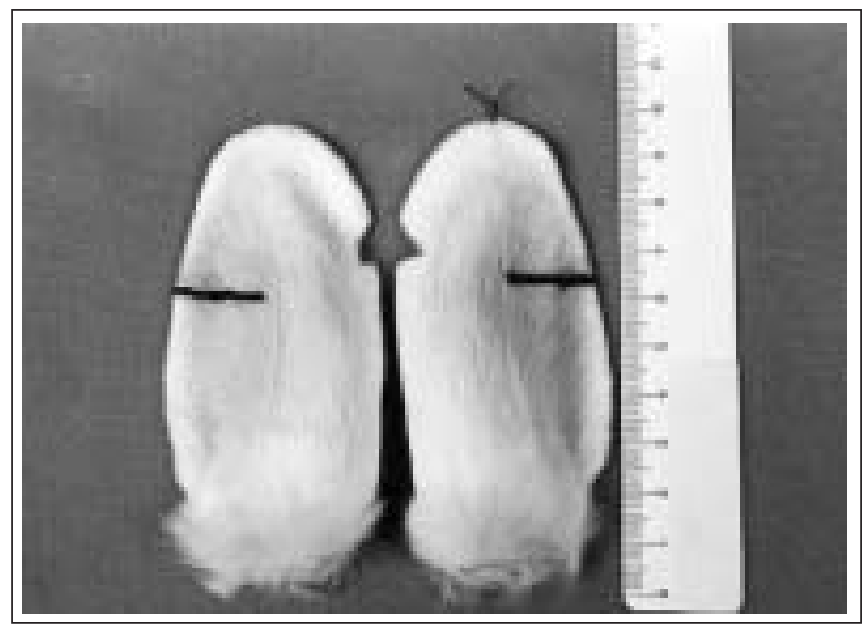

Figura 2 - Aurículas do Oryctolagus cuniculus, Animal n 5 , do Grupo Controle, Subgrupo 6, após a Eutanásia, em que se observa um corte triangular na parte rostral das aurículas, ponto com fio de algodão 000 na parte distal da aurícula direita e demarcação dos locais das venopunções

\section{RESULTADOS}

Para se estudar a presença de trombose, foi realizada a análise estatística em duas etapas.

Na primeira, foi utilizado o teste do Qui-quadrado para tabelas $2 \times \mathrm{N}$ para comparar os períodos de eutanásia de $6 \mathrm{~h}, 12 \mathrm{~h}$ e $24 \mathrm{~h}$, para cada um dos grupos (Tenoxicam $/ \mathrm{NaCl}$ a $0,9 \%$, Tenoxicam/Diluente e Cloreto de Sódio a 0,9\%) separadamente, não se observando diferenças significantes entre os períodos com relação às presenças de trombose.

$\mathrm{Na}$ segunda, foi utilizado o mesmo teste para comparar as presenças do total de trombose dos grupos Tenoxicam $/ \mathrm{NaCl}$ a 0,9\%, Tenoxicam/Diluente e Cloreto de Sódio a 0,9\%, independentemente do período de eutanásia, observando-se que as porcentagens de trombose dos grupos Tenoxicam $/ \mathrm{NaCl}$ a $0,9 \%$ e Tenoxicam/Diluente foram significantemente maiores do que as observadas no grupo Cloreto de Sódio a $0,9 \%$.

Os resultados estão contidos na tabela I, expressos nas figuras 3 e 4 e ilustrados na figura 5 .

\section{DISCUSSÃO}

No que diz respeito à trombose, independentemente da causa da agressão, a lesão endotelial tem uma poderosa influência trombogênica. Quando ocorre lesão da célula endotelial, o sangue entra em contato com o tecido colágeno adjacente, que induz a agregação de plaquetas. Aseguir, a fibrina forma uma rede fibrosa tridimensional que aprisiona eritrócitos, leucócitos e plaquetas, originando trombos, que podem crescer e até obliterar o fluxo sangüíneo ${ }^{3}$.

O fator de ativação das plaquetas, o mais potente agente ulcerogênico, pode ser outra causa para o aumento da permeabilidade intestinal, por indução de trocas na vascularização da mucosa ${ }^{4}$ ou por sua ação nos neutrófilos ${ }^{5}$. Sua produção local pode ser estimulada pelos antiinflamatórios não-esteróides, diretamente ou pelo desenvolvimento de uma resposta inflamatória ${ }^{6}$.

Nygard e col. observaram, em culturas com células endoteliais de veias umbilicais humanas expostas à indometacina, que a síntese de eicosanóides antitrombóticos era totalmente inibida por este antiinflamatório não-esteróide, levando os

Tabela I - Coelhos dos Grupos Experimento (Tenoxicam/ $\mathrm{NaCl}$ a 0,9\% e Tenoxicam/Diluente) e Controle (Cloreto de Sódio a $0,9 \%$ ), Segundo o Tempo de Observação (6 h, 12 h e 24 h) e a Presença de Trombose nas Veias Auriculares

\begin{tabular}{|c|c|c|c|c|c|c|c|c|c|c|c|c|}
\hline \multicolumn{13}{|c|}{ Trombose } \\
\hline \multirow[t]{2}{*}{ Obs. } & \multicolumn{4}{|c|}{ Experimento-Tenoxicam/ $\mathrm{NaCl}$ a $0,9 \%$} & \multicolumn{4}{|c|}{ Experimento - Tenoxicam/Diluente } & \multicolumn{4}{|c|}{ Controle - Cloreto de Sódio a $0,9 \%$} \\
\hline & Presente & Ausente & Total & $\%$ pres. & Presente & Ausente & Total & $\%$ pres. & Presente & Ausente & Total & $\%$ pres. \\
\hline $6 \mathrm{~h}$ & 2 & 10 & 12 & 16,7 & 3 & 9 & 12 & 25,0 & 0 & 12 & 12 & 0,0 \\
\hline $12 \mathrm{~h}$ & 2 & 10 & 12 & 16,7 & 2 & 10 & 12 & 16,7 & 0 & 12 & 12 & 0,0 \\
\hline $24 \mathrm{~h}$ & 4 & 8 & 12 & 33,3 & 2 & 10 & 12 & 16,7 & 0 & 12 & 12 & 0,0 \\
\hline Total & 8 & 28 & 36 & 22,2 & 7 & 29 & 36 & 19,4 & 0 & 36 & 36 & 0,0 \\
\hline
\end{tabular}

Teste do Qui-quadrado ( $6 \mathrm{~h} \times 12 \mathrm{~h} \times 24 \mathrm{~h}$, para cada grupo separadamente). Sem diferença estatística significante

Teste do Qui-quadrado [Tenoxicam/NaCl a 0,9\% x Tenoxicam/Diluente x Cloreto de Sódio a 0,9\%, independente do período de observação (6 h+12 h+24 h)] Diferença estatística significante com $p<0,05$ 


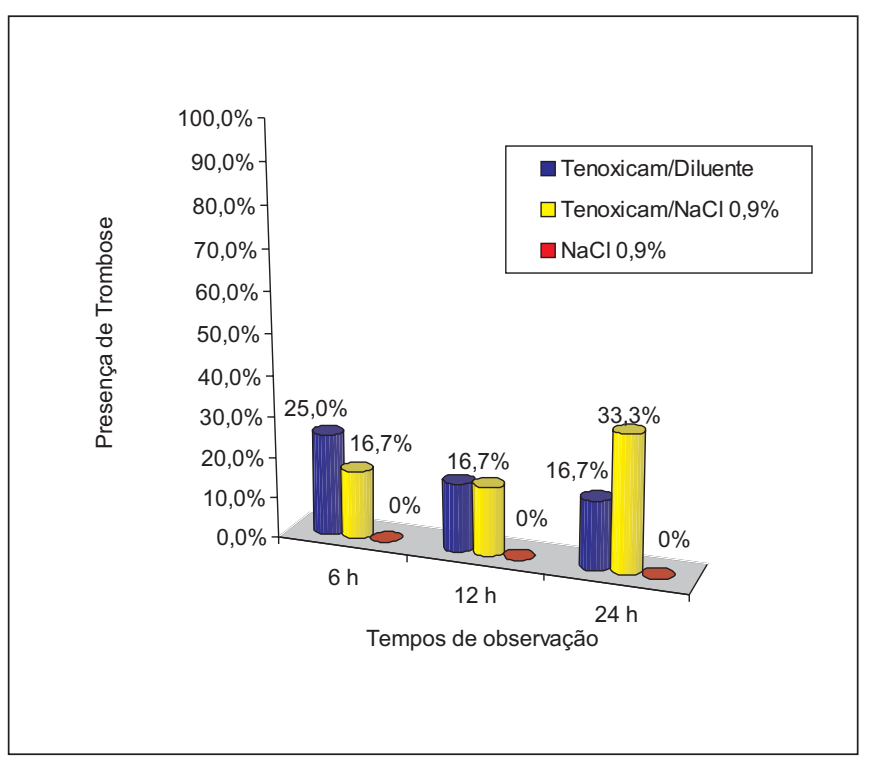

Figura 3 - Porcentagem de Veias com Presença de Trombose, nos grupos Controle $(\mathrm{NaCl}$ a $0,9 \%$ ) e Experimento (Tenoxicam/Diluente ou Tenoxicam/ $\mathrm{NaCl}$ a 0,9\%), nos Tempos de Observação de $6 \mathrm{~h}, 12 \mathrm{~h}$ e $24 \mathrm{~h}$

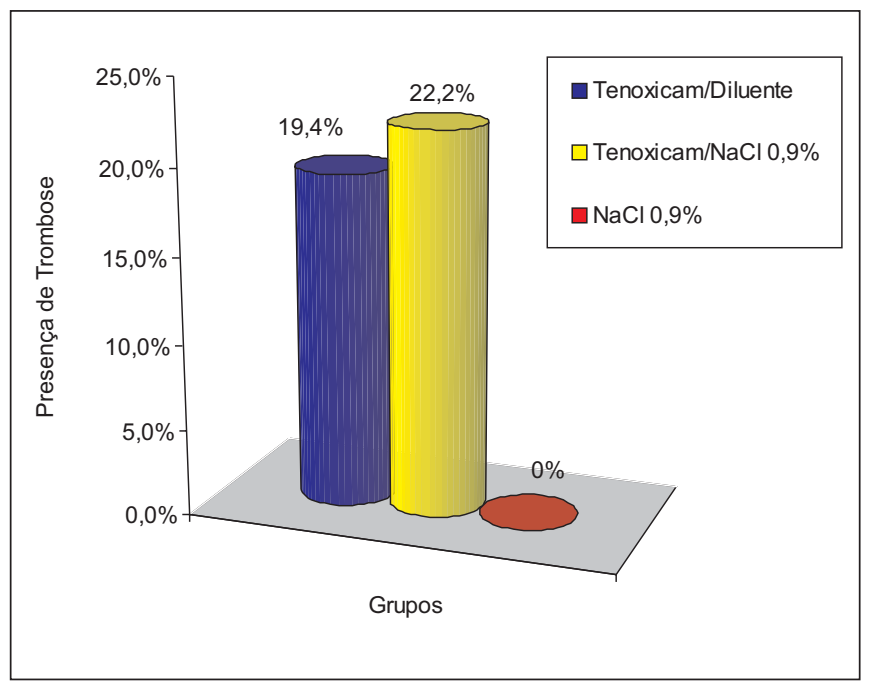

Figura 4 - Porcentagem de Veias com Presença de Trombose, nos Grupos Experimento (Tenoxicam/Diluente ou Tenoxicam/ $/ \mathrm{NaCl}$ a $0,9 \%)$ e Controle $(\mathrm{NaCl}$ a $0,9 \%)$, Independente dos Tempos de Observação

autores a concluírem que a indometacina induz atividade pró-coagulante nesta cultura de células ${ }^{1}$.

Schnaider e col., estudando as veias auriculares caudais de coelhos (Oryctolagus cuniculus), após a administração de tenoxicam com seu diluente comercial, observaram a presença de trombose em $19,4 \%$ das veias ${ }^{2}$.

No trabalho realizado por Nygard e col., em culturas com céIulas endoteliais de veias umbilicais humanas, a resposta da atividade pró-coagulante foi tempo-dependente ${ }^{1}$, ao passo que no trabalho realizado por Schnaider e col., em coelhos, não foram constatadas diferenças estatísticas significantes entre os tempos de avaliação ${ }^{2}$.

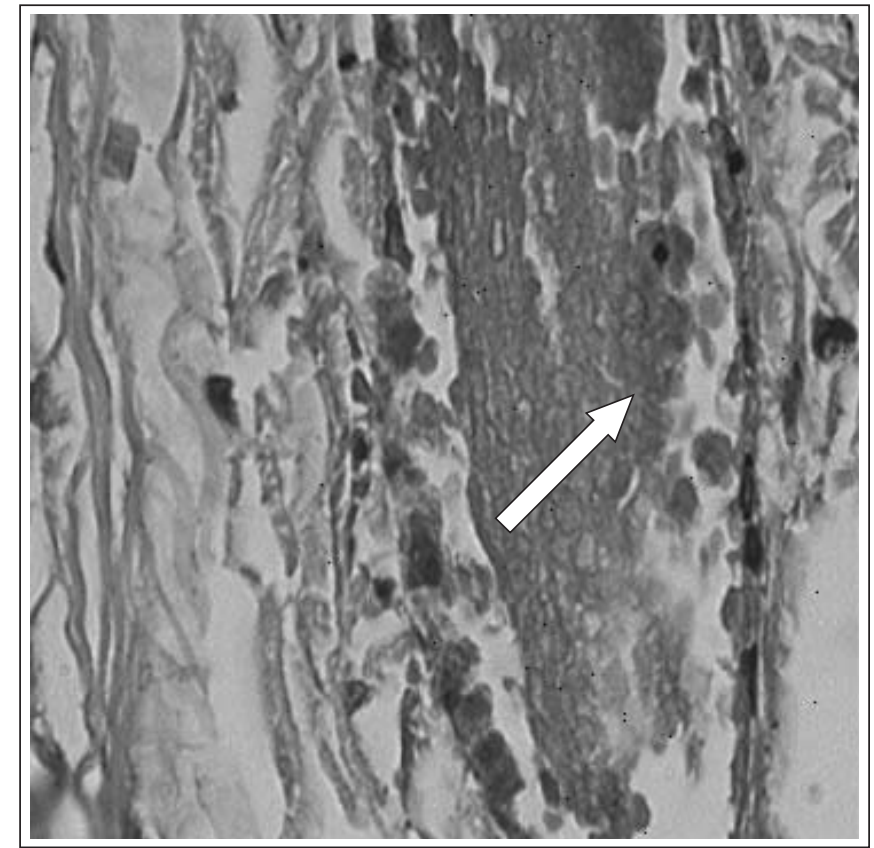

Figura 5 - Trombose na Veia Auricular Direita do Oryctolagus cuniculus, Animal $n^{\circ} 4$, do Grupo Experimento (Tenoxicam $/ \mathrm{NaCl}$ a $0,9 \%$ ), Subgrupo 24 , com Aumento de 400 Vezes

Apenas a disfunção endotelial, sem exposição do colágeno da camada íntima, é suficiente para promover a trombose. $O$ endotélio vascular, normalmente, proporciona uma superfície não-trombogênica devido à presença de sulfato de heparan de superfície, um glicosaminoglicano relacionado com a heparina, que, a exemplo desta última, é um co-fator da antitrombina III. Deste modo, o endotélio desempenha função essencial na prevenção da ativação plaquetária e coagulação intravascular. O endotélio, sendo agredido, induz a inativação de heparan e a ativação do fator tecidual, deixando de produzir óxido nítrico e prostaciclina, passando então a produzir endotelina. Os dois primeiros, além de vasodilatadores, também inibem a agregação plaquetária e a fixação da fibrina no trombo branco, enquanto que a endotelina é vasoconstritora e estimula a agregação plaquetária e a cascata da coagulação ${ }^{7}$.

Pode-se explicar a presença de trombose pela inibição de eicosanóides antitrombóticos induzindo atividade pró-coagulante. Seria uma via alternativa à inibição das ciclooxigenases, ocorrendo produção local do fator de ativação das plaquetas ou produção local de leucotrienos.

Outra possibilidade, que poderia explicar a trombose, ocorrida após a administração do antiinflamatório não-esteróide, seria o $\mathrm{pH}$ da solução e sua relação com o transporte do fármaco na membrana da célula. Aágua bidestilada apresenta pH de 6,34 , o cloreto de sódio a $0,9 \%$ de 5,67 , o tenoxicam/diluente de 8,49 e o tenoxicam $/ \mathrm{NaCl}$ a $0,9 \%$ de 8,40 . As membranas celulares apresentam superfícies polarizadas e hidrofílicas, com glicoproteínas receptoras e uma grande variedade de proteínas em sua estrutura, como a transmembrana. O peso molecular, o estado físico-químico e a solubilida- 
de de um fármaco influenciam sua absorção. As substâncias lipofílicas e com $\mathrm{pH}$ próximo à neutralidade penetram na membrana com maior facilidade. O tenoxicam, devido ao $\mathrm{pH}$ alcalino da solução, seria transportado na membrana provavelmente auxiliado por uma proteína transmembrana, induzindo disfunção endotelial, diretamente ou por meio de substâncias reativas formadas durante o metabolismo.

Neste estudo, foi observada a presença de trombose no grupo Experimento, tanto após a administração do tenoxicam com água bidestilada $(19,4 \%)$ quanto a do tenoxicam com cloreto de sódio a 0,9\% (22,2\%). Comparando-se a presença de trombose nos períodos de eutanásia de $6 \mathrm{~h}, 12 \mathrm{~h}$ e $24 \mathrm{~h}$, para cada um dos grupos separadamente, não foram encontradas diferenças significantes de trombose entre os períodos, conforme pode ser observado na tabela I.

Conclui-se então que o tenoxicam, tanto com água bidestilada quanto com cloreto de sódio a $0,9 \%$, produz trombose nas veias em que foi administrado.

\section{Histopathologic Study on the Effects of Tenoxicam with Bidistilled Water or with $0.9 \%$ Sodium Chloride in Rabbits Venous Endothelium}

Taylor Brandão Schnaider, TSA, M.D., Alcino Lázaro da Silva, M.D., Míriam de Fátima Brasil Engelman, M.D., Yara Juliano, M.D., Neil Ferreira Novo, M.D., Gabrielle Sormanti Schnaider, M.D., Caroline Sormanti Schnaider, M.D.

\section{INTRODUCTION}

After observing in our anesthesiology clinic some patients referring signs and symptoms of vasculytis (pain, heat, redness) after direct intravenous tenoxicam with bidistilled water (without commercial diluent), we have searched the literature but were unable to find studies on this event in humans. Nygard et al., as a consequence of the association of non-steroid anti-inflammatory drugs and intestinal microvascular injuries, have studied coagulation abnormalities in human umbilical vein endothelial cells exposed to indomethacin, bacterial lipopolysaccharides or their association, after $1 \mathrm{~h}, 6 \mathrm{~h}, 12 \mathrm{~h}$ and $24 \mathrm{~h}$. The authors have found the following results: lipolysaccharides have significantly induced procoagulant activity at $6 \mathrm{~h}, 12 \mathrm{~h}$ and $24 \mathrm{~h}$, with a significant increase in 6-keto-PGF $F_{1 \alpha}$, while the increase in $\mathrm{PGE}_{2}$ production was less pronounced; with indomethacin alone, the procoagulant activity was time-dependent; with Indomethacin and lipopolysaccharides, procoagulant response was more significant than that produced by cultures with just indomethacin or lipopolysaccharides ${ }^{1}$.

Schnaider et al., in experimental studies with rabbits, have hystopathologically evaluated the presence or absence of thrombosis in the venous endothelium after the administrati- on of tenoxicam with its commercial diluent or its diluent alone. Authors have found $19.4 \%$ thrombosis in both case ${ }^{2}$, but this study has raised a question: was thrombosis caused by tenoxicam or by bidistilled water used as a diluent.

This study aimed at hystopathologically evaluating whether tenoxicam with $0.9 \% \mathrm{NaCl}$ would change rabbits venous endothelium similarly to what is seen when this anti-inflammatory is associated to bidistilled water.

\section{METHODS}

After the Animal Research Ethical Committee approval, 90 white, New Zealand, male rabbits (Oryctolagus cuniculus), aged above 10 weeks, weighing 2000 to 3500 grams, were randomly distributed in two groups: Experimental and Control. The Experimental group was subdivided in 3 subgroups of 24 rabbits each, which were called E6, E12 and E24, corresponding respectively to $6 \mathrm{~h}, 12 \mathrm{~h}$ and $24 \mathrm{~h}$ of observation and randomly receiving tenoxicam/diluent or tenoxi$\mathrm{cam} / 0.9 \% \mathrm{NaCl}$ in the right or left caudal auricular vein. The Control group was also subdivided in 3 subgroups of 6 rabbits each, which were called subgroups C6, C12 and C24, corresponding respectively to $6 \mathrm{~h}, 12 \mathrm{~h}$ and $24 \mathrm{~h}$ of observation and receiving $0.9 \% \mathrm{NaCl}$ in the right or left caudal auricular vein. There were no food and water restrictions until beginning of the experiment, when animals were weighed and anesthetized with muscular $1 \%$ acepromazine maleate $\left(2 \mathrm{mg}^{\mathrm{kg}} \mathrm{kg}^{-1}\right)$, ketamine hyodrochloride $\left(35 \mathrm{mg} . \mathrm{kg}^{-1}\right)$ and $2 \%$ xilazine hydrochloride $\left(5 \mathrm{mg} \cdot \mathrm{kg}^{-1}\right)$ in the gluteal region.

Ten minutes after anesthesia, animals were placed in the supine position and chest and pelvic limbs were tied with strings to the table's edges.

After 15 minutes, anesthetic depth was tested by response of right pelvic limb to a painful stimulus in the skin fold between digits II and III.

Next, antisepsis was performed with $70 \%$ alcohol and animals were covered with sterilized drapes. Right and left caudal auricular veins were punctured with a $27 \mathrm{G}$ butterfly needle. The following drugs were randomly injected: tenoxi-

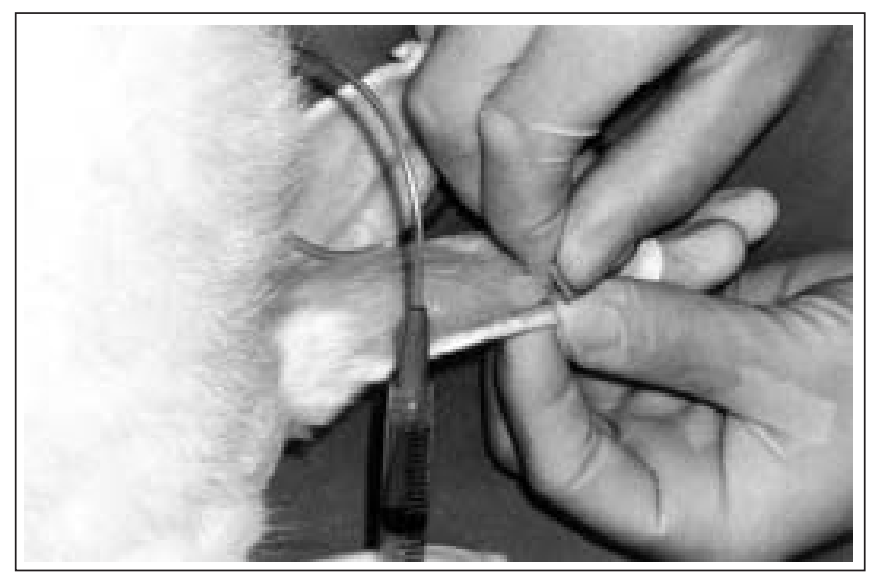

Figure 1 - Caudal Auricular Vein Puncture with 27G Butterfly Needle for Drug Administration 
cam/diluent or tenoxicam $/ 0.9 \% \mathrm{NaCl}$ in the Experimental group and $0.9 \% \mathrm{NaCl}$ in the Control group (Figure 1). Puncture sites were then marked with a "Texta fine line 7000 " pen. Substances $(2 \mathrm{ml})$ were previously packaged in $3 \mathrm{ml}$ syringes and were injected at the speed of $1 \mathrm{ml}$ for 30 seconds.

After the evolution time scheduled for each subgroup $(6 \mathrm{~h}, 12 \mathrm{~h}$ or $24 \mathrm{~h}$ ), animals were again anesthetized by the same technique used in the experiment, and sacrificed with $3 \mathrm{ml}$ intracardiac $10 \%$ potassium chloride. Next, a triangular cut was performed at the auricles rostral portion and a stitch, with 000 cotton thread was placed at the distal portion of the right auricle. Auricles were then resected at their base and placed in $10 \%$ neuter buffered formalin after macroscopic observation (Figure 2). Containers with surgical specimens were identified by group, subgroup and animal's number. Specimens were place in wax and 5 sections with 5 micrometers of width each were performed at $1 \mathrm{~cm}$ from the puncture site, proximal to the auricle, using a rotating mocrotome with disposable blade (Leica RM-2025). Sections were then placed in thin plates, died with hematoxylin-eosin ( $\mathrm{H}-\mathrm{E})$ and covered by glass slides. Plates were analyzed by optical microscopy (Lambda LQT-2) with original enhancements of 40,100 and 400 times,

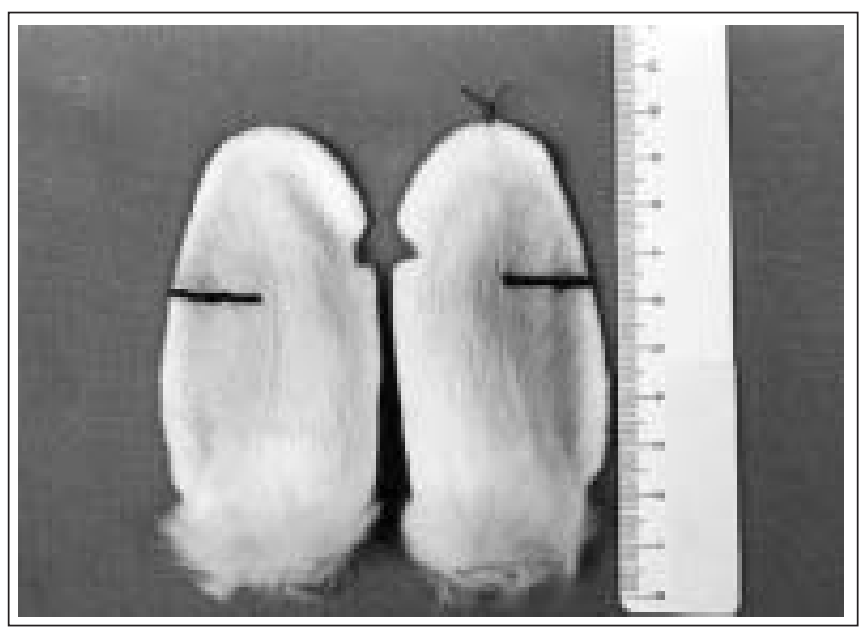

Figure 2 - Auricles from Oryctolagus cuniculus, Rabbit $\mathrm{n}^{\circ}$ 5, from Control Group, Subgroup 6, after euthanasia, were it is possible to observe a triangular section at auricles rostral side, stitch with cotton thread 000 at right auricle distal portion and venous puncture sites demarcation digitized (Samsung SHC-410 NAD Camera) and written in 3 $1 / 2$ diskettes.

Chi-square partition test for $2 \mathrm{xN}$ tables was used to compare, for each group, $6 \mathrm{~h}, 12 \mathrm{~h}$ and $24 \mathrm{~h}$ of euthanasia with regards to thrombosis. The same test was applied to compare total thrombosis incidence in tenoxicam/diluent and tenoxicam $/ 0.9 \% \mathrm{NaCl}$ Control groups, regardless of the euthanasia time. Significance level was 0.05 or $5 \%(\alpha \leq 0.05)$ for all tests and a bold star $\left({ }^{*}\right)$ was used to highlight significant values.

\section{RESULTS}

To analyze the incidence of thrombosis, statistical analysis was performed in two stages. In the first stage, Chi-square test for $2 \mathrm{xN}$ tables was used to compare euthanasia periods of $6 \mathrm{~h}, 12 \mathrm{~h}$ or $24 \mathrm{~h}$, for each group (Tenoxicam $/ 0.9 \% \mathrm{NaCl}$, Tenoxicam/Diluent and $0.9 \% \mathrm{NaCl}$ ) without significant differences among periods.

In the second stage, the same test was used to compare total thrombosis of Tenoxicam $/ 0.9 \% \mathrm{NaCl}$ and Tenoxicam/Diluent and $0.9 \% \mathrm{NaCl}$ groups, regardless of the euthanasia period, observing that Tenoxicam $/ 0.9 \% \mathrm{NaCl}$ and Tenoxicam/Diluent groups thrombosis incidence was significantly higher than in the $0.9 \% \mathrm{NaCl}$ group.

Results are shown in table I, expressed in figures 3 and 4 and illustrated in figure 5 .

\section{DISCUSSION}

Regardless of the triggering aggression, endothelial injuries suffer a major thormbogenic influence. When there is an endothelial cell injury, blood gets in touch with the adjacent collagen tissue which induces platelet aggregation. Next, fribin forms a tridimensional fibrous net which traps erythrocytes, leucocytes and platelets originating thrombi, which may grow and even block blood flow ${ }^{3}$.

Platelets activation factor, most potent ulcerogenic agent, may be another cause for increased intestinal patency, by mucosal vascularization exchanges induction ${ }^{4}$ or by acting on neutrophils ${ }^{5}$. Its local production may be directly stimula-

Table I - Experimental Groups (Tenoxicam/0.9\% NaCl and Tenoxicam/Diluent) and Control Group Rabbits (0.9\% sodium chloride), According to the Observation Period (6 h, $12 \mathrm{~h}$ and $24 \mathrm{~h}$ ) and the Presence of Thrombosis in Auricular Veins

\begin{tabular}{|c|c|c|c|c|c|c|c|c|c|c|c|c|}
\hline \multicolumn{13}{|c|}{ Thrombosis } \\
\hline \multirow[t]{2}{*}{ Obs. } & \multicolumn{4}{|c|}{ Experimental-Tenoxicam $/ 0.9 \% \mathrm{NaCl}$} & \multicolumn{4}{|c|}{ Experimental -Tenoxicam/Diluent } & \multicolumn{4}{|c|}{ Control - $0.9 \%$ Sodium Chloride } \\
\hline & Present & Absent & Total & $\%$ pres. & Present & Absent & Total & $\%$ pres. & Present & Absent & Total & $\%$ pres. \\
\hline $12 \mathrm{~h}$ & 2 & 10 & 12 & 16.7 & 2 & 10 & 12 & 16.7 & 0 & 12 & 12 & 0.0 \\
\hline $24 \mathrm{~h}$ & 4 & 8 & 12 & 33.3 & 2 & 10 & 12 & 16.7 & 0 & 12 & 12 & 0.0 \\
\hline Total & 8 & 28 & 36 & 22.2 & 7 & 29 & 36 & 19.4 & 0 & 36 & 36 & 0.0 \\
\hline
\end{tabular}

Chi-square test $(6 \mathrm{~h} \times 12 \mathrm{~h} \times 24 \mathrm{~h}$, for each group separately). Without statistical significance

Chi-square test [Tenoxicam/0.9\% NaCl x Tenoxicam/Diluent x $0.9 \%$ Sodium Chloride, regardless of observation period $(6 \mathrm{~h}+12 \mathrm{~h}+24 \mathrm{~h})$ ]. Statistically significant difference; $p<0.05$ 


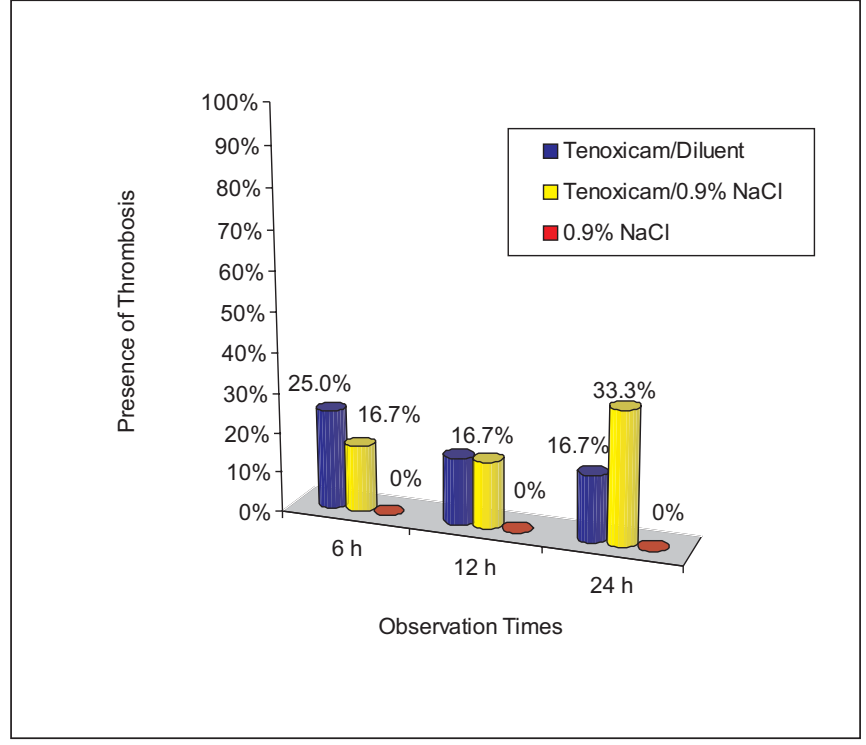

Figure 3 - Percentage of Thrombotic Veins in Control ( $\mathrm{NaCl}$ a $0.9 \%)$ and Experimental groups (Tenoxicam/Diluent or Tenoxicam $/ 0.9 \% \mathrm{NaCl}$ ), in Observation Times of $6 \mathrm{~h}, 12 \mathrm{~h}$ e $24 \mathrm{~h}$

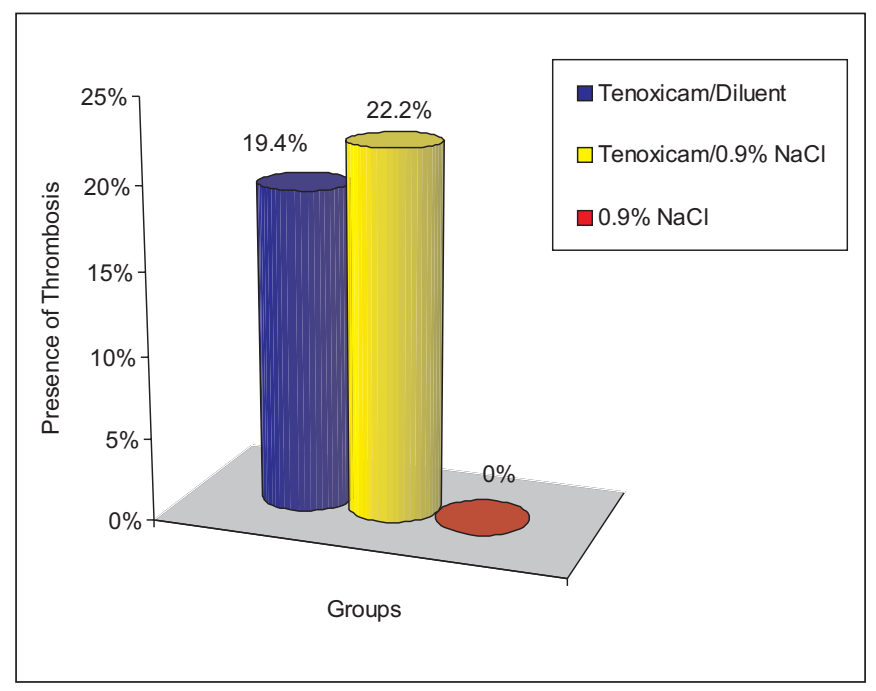

Figure 4 - Percentage of Thrombotic Veins in Control ( $\mathrm{NaCl}$ a $0.9 \%)$ and Experimental groups (Tenoxicam/Diluent or Tenoxicam $/ 0.9 \% \mathrm{NaCl}$ ), regardless of Observation Times

ted by NSAIDs or by the development of an inflammatory response ${ }^{6}$.

Nygard et al. have observed in human umbilical vein endothelial cells exposed to Indomethacin, that the synthesis of anti-thrombotic eicosanoids was totally inhibited by this NSAID, leading them to conclude that Indomethacin induces procoagulant activity in these cell cultures ${ }^{1}$.

Schnaider et al., studying caudal auricular veins of rabbits (Oryctolagus cuniculus) after tenoxicam administration with its commercial diluent, have observed the presence of thrombosis in $19.4 \%$ of veins ${ }^{2}$.

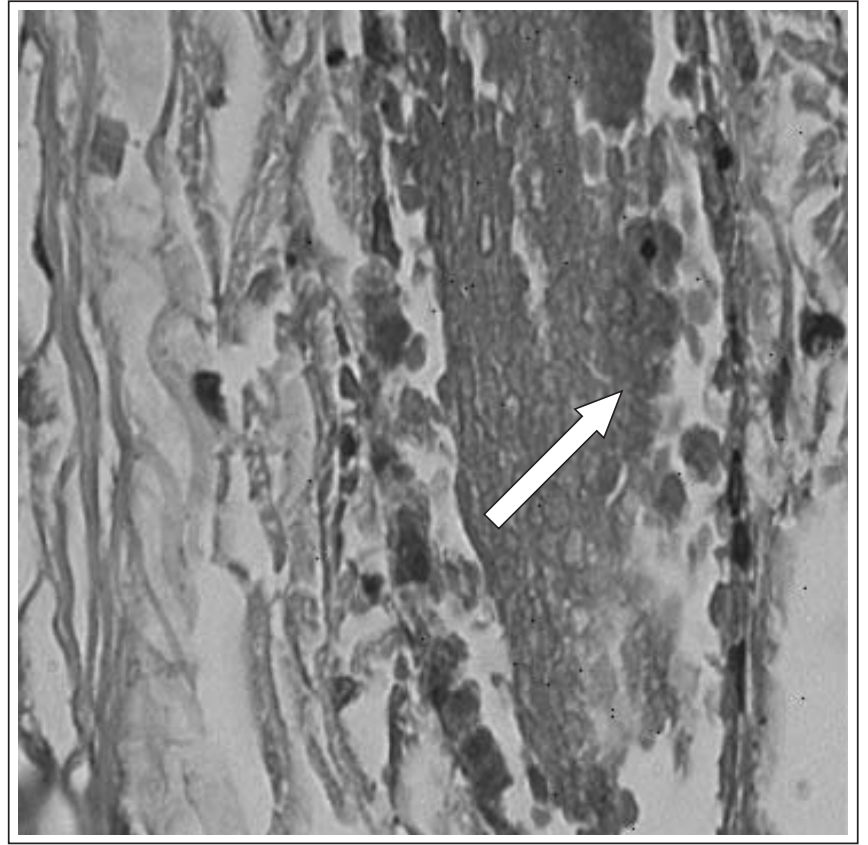

Figure 5 - Right Auricular Vein Thrombosis of Oryctolagus cuniculus, Animal $n^{\circ} 4$, of Experimental Group (Tenoxicam $/ 0.9 \%$ $\mathrm{NaCl}$ ), Subgroup 24, Enhanced 400 Times

In a study by Nygard et al. with human umbilical vein endothelial cell cultures, procoagulant activity was time-dependent ${ }^{1}$, whereas in the study by Schnaider et al., no statistically significant differences were found in rabbits among evaluation times $^{2}$.

The endothelial dysfunction alone, without exposure to the intima layer collagen, is enough to promote thrombosis. In general, the vascular endothelium has a non-thrombogenic surface due to the presence of surface heparan sulphate, a heparin-related glycosaminoglycan which, as heparin, is an anti-thrombin III co-factor. So, the endothelium plays an essential role in preventing platelet aggregation and intravascular coagulation. When aggressed, the endothelium induces heparan and tissue factor activation, no longer producing nitric oxide and prostacyclins, but producing endothelin. The two former agents, in addition to being vasodilators, also inhibit platelet aggregation and fibrin fixation to the white thrombus, while endothelin is vasoconstrictor and stimulates platelet aggregation and the coagulation cascade ${ }^{7}$.

Thrombosis may be explained by the inhibition of anti-thrombotic eucosanoids, thus inducing procoagulant activity. This would be an alternative pathway for cycloxygenase inhibition with local platelet activation factor or leucotriens production. Another hypothesis to explain thrombosis after NSAIDs administration would be the solution's $\mathrm{pH}$ and its relation with drug transportation in the cell membrane. Bidistilled water has a pH of $6.34,0.9 \%$ sodium chloride of 5.67 , tenoxicam/diluent of 8.49 and tenoxicam $/ 0.9 \% \mathrm{NaCl}$ of 8.40 . Cell membranes have polarized and hydrophilic surfaces with receptor glucoproteins and a wide variety of structural proteins, such as transmembrane. Drugs molecular weight, physical-che- 
mical properties and solubility may influence their absorption. Lipophylic solutions with a $\mathrm{pH}$ close to neutrality easily penetrate the membrane. Tenoxicam, due to its alkaline $\mathrm{pH}$, would be transported in the membrane, probably helped by a trasmembrane protein, directly inducing endothelial dysfunction or by means of reactive substances generated during metabolism.

In our study, thrombosis was observed in the Experimental group both after tenoxicam plus bidistilled water administration (19.4\%) and after tenoxicam plus $0.9 \% \mathrm{NaCl}(22.2 \%)$. In comparing the incidence of thrombosis in $6 \mathrm{~h}, 12 \mathrm{~h}$ and $24 \mathrm{~h}$ euthanasia periods for each group separately, no significant differences were found among periods, as shown in table I.

The conclusion was that tenoxicam, both with bidistilled water and $0.9 \%$ sodium chloride, produces thrombosis in the veins used for its administration.

\section{REFERÊNCIAS - REFERENCES}

01. Nygard G, Hudson M, Mazure G et al - Procoagulant and prothrombotic responses of human endothelium to indomethacin and endotoxin in vitro. Relevance to non-steroidal anti-inflammatory drug enteropathy. Scand J Gastroenterol, 1995;30:25-32.

02. Schnaider TB, Andrade CHV, Juliano $Y$ et al - Estudo histopatológico do efeito do tenoxicam e do seu diluente no endotélio venoso, em coelhos. Acta Cir Bras, 2000;15:118-123.

03. Cotran RS, Kumar V, Robbins S - Robbins Patologia Estrutural e Funcional, 5a Ed, Rio de Janeiro, Guanabara Koogan, 1996;84-109.

04. Whittle BJR, Morishita T, Ohya Y et al - Microvascular actions of platelet-activating factor on rat gastric mucosa and submucosa. Am J Physiol, 1986;251:G772-G778.

05. Wallace JL, Keenan CM, Granger DN - Gastric ulceration induced by nonsteroidal anti-inflammatory drugs is a neutrophil-dependent process. Am J Physiol, 1990;259: G462-G467.

06. Wallace JL - Lipid mediators of inflammation in gastric ulcer. Am J Physiol, 1990;21:G1-G11.

07. Rang HP, Dale MM, Ritter JM - Farmacologia, $2^{a}$ Ed, Rio de Janeiro, Guanabara Koogan, 2001;255-269.

\section{RESUMEN}

Schnaider TB, Silva AL, Engelman MFB, Juliano Y, Novo NF, Schnaider GS, Schnaider CS - Estudio Histopatológico del Efecto del Tenoxican con Agua Bidestilada o con Cloreto de Sodio a $0,9 \%$ en el Endotélio Venoso de Conejos

Justificativa y Objetivos - En estudio con células endoteliales de venas umbilicales humanas expuestas a la indometacina, fue observado aumento de la actividad pró-coagulante. Estudio en conejos comprobó la presencia de trombosis en las venas auriculares después de la administración de tenoxicam con su diluyente o de su diluyente aislado. No fueron encontrados estudios en la literatura consultada que hayan evaluado el endotélio venoso después de la administración de tenoxicam, en seres humanos. El objetivo de esta pesquisa fue evaluar se el tenoxicam con cloreto de sodio a 0,9\% ( $\mathrm{NaCl}$ a $0,9 \%$ ) provoca alteraciones en el endotélio venoso de conejos, como las observadas cuando asociado a su diluyente (agua bidestilada).

Método - Noventa conejos (2.000 - $3.500 \mathrm{~g})$ fueron distribuidos aleatoriamente en dos grupos: Control, con administración de $\mathrm{NaCl}$ a 0,9\%; Experimento, con tenoxicam (20 mg) asociado a agua bidestilada o al $\mathrm{NaCl}$ a 0,9\%. El volumen inyectado en los dos grupos fue constante de $2 \mathrm{ml}$. La anestesia fue inducida con maleato de acepromazina, clorhidrato de cetamina y clorhidrato de xilazina, siendo la punción de las venas auriculares caudales derecha e izquierda realizada con aguja tipo mariposa 27G. Los animales fueron mantenidos en el biotério por $6 h, 12 h$ e $24 h$, nuevamente anestesiados y sometidos a eutanasia, siendo entonces realizada exéresis de las aurículas en su base y posterior evaluación microscópica de las venas.

Resultados - Se observó trombosis en el grupo Experimento, en una porcentaje de $19,4 \%$ después de administración del tenoxicam con agua bidestilada y 22,2\% después administración del tenoxicam con $\mathrm{NaCl}$ a 0,9\%. En el grupo Control, en que fue inyectado, solamente $\mathrm{NaCl}$ a $0,9 \%$, ninguna de las venas presento trombosis.

Conclusiones - Los resultados encontrados permiten concluir que el tenoxicam, con agua bidestilada o con solución de cloreto de sodio a $0,9 \%$, produjo trombosis en las venas en que fue inyectado. 